I. Kubo and S.-S. Lee

Nagoya Math. J.

Vol. 95 (1984), 23-40

\title{
NON-LINEAR PREDICTORS OF TRANSFORMED STATIONARY PROCESSES
}

\author{
IZUMI KUBO AND SHEU-SAN LEE
}

\section{§1. Introduction}

One of the authors discussed the best non-linear predictor of the process $X(t)=f(U(t))$, which is obtained by transforming an OrnsteinUhlenbeck process $U(t)$ with a measurable function $f(u)$ (see [5]). We denote by $\mathscr{B}_{t}(X)$ the completion of the $\sigma$-field generated by $\{X(s, \omega) ; s \leq t\}$, and by $\widetilde{\mathscr{B}}_{t}(X)$ the completion of the $\sigma$-field generated by $\{X(s, \omega) ; s \geq t\}$. Assuming that $X(t)$ is square integrable, he defined the best non-linear predictor $\hat{X}(t ; \tau, \omega)$ of $X(t+\tau, \omega)$ for the given data $\{X(s, \omega) ; s \leq t\}$ by

$$
\hat{X}(t ; \tau) \equiv E\left[X(t+\tau) \mid \mathscr{B}_{t}(X)\right],
$$

which gives the least mean square error. It can be rewritten as

$$
\hat{X}(t ; \tau)=E\left[\left(T_{\tau}^{U} f\right)(U(t)) \mid \mathscr{B}_{t}(X)\right] \text { a.s., }
$$

where $T_{\tau}^{U}, \tau>0$, is the semi-group of the Ornstein-Uhlenbeck process $U(t)$. He has shown that $\mathscr{B}_{t}(X)=\mathscr{B}_{t}(U)$ holds in many cases and that algorithms determining the value of $U(t, \omega)$ by the observed data can be given.

In this paper, we shall see that his methods can be applied to more general base processes $\{V(t, \omega), t \in R\}$. Throughout this paper, we assume that each base process $\{V(t)\}$ is a strictly stationary process with continuous paths defined on a probability space $(\Omega, \mathscr{B}, P)$ and that $V(t, \omega)$ takes its values in an interval $(\ell, r)$ with $-\infty \leq \ell<r \leq \infty$, and satisfies

$$
\varliminf_{t \rightarrow-\infty} V(t, \omega)=\ell \text { and } \varlimsup_{t \rightarrow-\infty} V(t, \omega)=r \text { a.s. }
$$

Let $m(d v)$ be the distribution of $V(t)$ for each $t$ and let $f(v)$ be a function in $L^{2}(d m)$. Then the transformed process $X(t, \omega) \equiv f(V(t, \omega)), t \in R$, is a strictly stationary process with the variance $\int_{\ell}^{r} f(v)^{2} d m(v)-\left(\int_{\ell}^{r} f(v) d m(v)\right)^{2}$.

Received September 16, 1982. 
The best non-linear predictor $\hat{X}(t ; \tau, \omega)$ of $X(t+\tau, \omega)$ for the observed data $\{X(s, \omega) ; s \leq t\}$ is defined by

$$
\hat{X}(t ; \tau) \equiv E\left[f(V(t+\tau)) \mid \mathscr{B}_{t}(X)\right]=E\left[E\left[f(V(t+\tau)) \mid \mathscr{B}_{t}(V)\right] \mid \mathscr{B}_{t}(X)\right],
$$

which gives the least mean square error. Our problem is to give an algorithm to determine $\hat{X}(t ; \tau, \omega)$ in terms of the observed data $\{X(s, \omega)$; $s \leq t\}$.

In Section 2, we shall discuss the case that the base process $V(t)$ satisfies the local limit theorem

$$
\varlimsup_{h \rightarrow 0} \frac{|V(t+h, \omega)-V(t, \omega)|}{\gamma(h)}=1 \text { a.s. } \quad \text { for any } t \in R,
$$

with a suitable $\gamma(h)$ of $\gamma(h)=o(1)$. If $f(v)$ is a piecewise monotone and differentiable function without any local symmetry (see Definition 2.3), then we can show $\mathscr{B}_{t}(X)=\mathscr{B}_{t}(V)$ and $\hat{X}(t, \tau)=E\left[f(V(t+\tau)) \mid \mathscr{B}_{t}(V)\right]$ (see Theorems 2.4 and 2.5). If $f(v)$ has a single bottom without peaks (see Definition 2.1) additionally, then we can give an algorithm to determine $V(t)$ by the observed data $\{X(s) ; s \leq t\}$. The condition (1.5) is weakened as (2.5).

In Section 3, we shall discuss the case that the base process $V(t)$ is a diffusion. The results are very similar to those in [5].

In Section 4, we shall discuss the case that $V(t)$ has smooth sample paths. If $V(t)$ has the property that for any fixed $v$

$$
P\left(\left\{\omega: \exists t \in R, V(t, \omega)=v, V^{\prime}(t, \omega)=0\right\}\right)=0
$$

holds, then we have a finer result (see Theorem 4.3).

In Section 5, stationary Gaussian processes will be discussed. In particular, we give precise results for double Markov, stationary Gaussian processes (see Theorem 5.2).

Acknowledgement. The authors would like to express their hearty thanks to Professor T. Hida and the members of Seminar on Probability in Nagoya for valuable suggestions and encouragements.

\section{§2. Prediction appealing to local limit theorems}

Now we are going to discuss the best non-linear prediction problem of the transformed process $X(t)=f(V(t))$, assuming that the base process $V(t)$ satisfies the conditions already stated in Section 1 . We restrict the 
class of functions $f(v)$ defined on the range $(\ell, r)$ of $V(t)$.

Definition 2.1. A function $f(v)$ on $(\ell, r)$ is simply said to be piecewise monotone, if $f(v)$ is a continuous function*) with extremum points $v_{1}<v_{2}<\cdots<v_{L}$ finite in number and if $f(v)$ is strictly monotone on each interval $\left(v_{j}, v_{j+1}\right), 0 \leq j \leq L$, with $v_{0} \equiv \ell$ and $v_{L+1} \equiv r$. We call $v_{j}$ a peak (resp. a bottom), if $v_{j}$ is a maximal (resp. minimal) point of $f(v)$.

Throughout this paper, we assume that $f(v)$ is piecewise monotone and piecewise continuously differentiable. Additionally, we assume that $f(v)$ belongs to $L^{2}(d m)$.

Let $\left\{v_{1}, \cdots, v_{L}\right\}$ be the set of all extremum points of $f(v)$. Set

$$
\begin{aligned}
& I_{j} \equiv\left(v_{j}, v_{j+1}\right], \quad 0 \leq j \leq L-1, \\
& I_{L} \equiv\left(v_{L}, v_{L+1}\right) \quad \text { and } \quad \bar{I}_{j} \equiv\left[v_{j}, v_{j+1}\right], \quad 0 \leq j \leq L .
\end{aligned}
$$

Put $f\left(v_{0}\right) \equiv f(\ell+0)$ and $f\left(v_{L+1}\right) \equiv f(r-0)$. Define $f_{\jmath}^{-1}(h)$ by

$$
f_{j}^{-1}(h) \equiv\left\{\begin{array}{lll}
v & \text { if } f(v)=h & \text { and } \quad v \in \bar{I}_{j}, \\
\Delta & \text { if } f(v) \neq h & \text { for any } v \in \bar{I}_{j},
\end{array}\right.
$$

with an extra point 4 . Define a process $j(s, \omega)$ by

$$
j(s, \omega) \equiv j \quad \text { if } V(s, \omega) \in I_{j}, 0 \leq j \leq L .
$$

Obviously we have

$$
V(s, \omega)=f_{j(s, \omega)}^{-1}(X(s, \omega)) .
$$

Firstly, we discuss $V(t)$ satisfying the following local limit theorem. Let $\gamma(h)$ be a continuous even function defined in a neighbourhood of $h=0$ with $\gamma(h)=o(1)$ as $h \rightarrow 0$. Assume that

$$
\varlimsup_{h \rightarrow 0} \frac{V(t+h, \omega)-V(t, \omega)}{\gamma(h)}=1 \text { a.s. }
$$

Then the following lemma is obviously proved, while useful.

Lemma 2.2. (i) Let $D(f)$ be the set of all $v$ at which $f(v)$ is differentiable. Then

$$
O^{\gamma}(X ; t, \omega) \equiv \varlimsup_{h \rightarrow 0} \frac{|X(t+h, \omega)-X(t, \omega)|}{\gamma(h)}=\mid f^{\prime}(V(t, \omega))
$$

*) The continuity of $f(v)$ is not essential for our discussions. But we assume it for simplicity. 
for a.e. $\omega$ in $\{\omega ; V(t, \omega) \in D(f)\}$.

(ii) If $T(\omega)$ is a random variable taking its values only in rational numbers, then

$$
O^{r}(X ; T(\omega), \omega)=\varlimsup_{h \rightarrow 0} \frac{|X(T(\omega)+h, \omega)-X(T(\omega), \omega)|}{\gamma(h)} \text { a.s. }
$$

Let us observe the two dimensional process $X(t, \omega) \equiv(X(t, \omega)$, $\left.O^{r}(x ; t, \omega)\right) \equiv\left(f(V(t, \omega)),\left|f^{\prime}(V(t, \omega))\right|\right)$, which is $\mathscr{B}_{t}(X)$-measurable. Obviously, $\mathscr{B}_{t}(X)=\mathscr{B}_{t}(V)$ holds, if $f(v)$ and $\left|f^{\prime}(v)\right|$ separate each point of the interval $(\ell, r)$. We shall give weaker conditions for $\mathscr{B}_{t}(X)=\mathscr{B}_{t}(V)$ and algorithms to determine $V(t)$ by the observed data $\{X(s) ; s \leq t\}$. For the purpose, we introduce a terminology.

Definition 2.3. We say that $f(v)$ has a local symmetry with respect to $v^{*}$, if $f\left(v^{*}+v\right)=f\left(v^{*}-v\right)$ holds in a neighbourhood of $v=0$. We say that $f(v)$ has a global symmetry with respect to $v^{*}$, if $f\left(v^{*}+v\right)=f\left(v^{*}-v\right)$ holds for any $v$ with $\ell<v^{*}+v<r$.

Now we suppose that $f(v)$ has a unique bottom $v_{1}$ without peaks and that it does not have any local symmetry with respect to $v_{1}$. Let $G(f)$ be the interior of the set of all $h$ 's such that $f_{0}^{-1}(h) \neq f_{1}^{-1}(h)$ holds, and $-f^{\prime}\left(f_{0}^{-1}(h)\right) \neq f^{\prime}\left(f_{1}^{-1}(h)\right)$ holds whenever both $f_{0}^{-1}(h)$ and $f_{1}^{-1}(h)$ are in $D(f)$. Then $G(f)$ is non empty. Define random times $T_{j}(t, \omega), j=0,1$, by

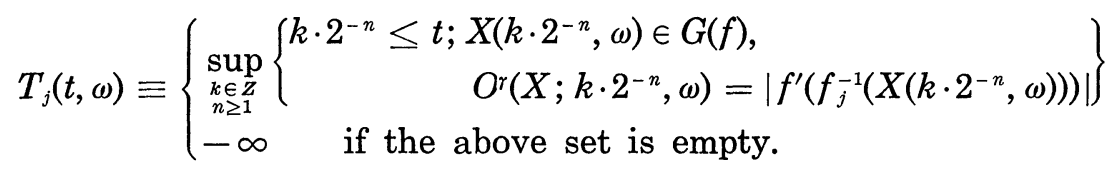

By Lemma 2.2 and the assumption (1.3), we can see that $T_{j}(t, \omega)>-\infty$ a.s. for $j=0,1$. We have obviously that

$$
j(t, \omega)= \begin{cases}0 & \text { if } T_{0}(t, \omega) \geq T_{1}(t, \omega), \\ 1 & \text { if } T_{0}(t, \omega)<T_{1}(t, \omega)\end{cases}
$$

almost surely. Moreover $T_{1-j}(t, \omega)$ is the time when $V(s, \omega)(s \leq t)$ passes through $v_{1}$ lastly, if $j(t, \omega)=j$. Thus we have

Theorem 2.4. If $V(t)$ satisfies the local limit theorem (2.3) and if $f(v)$ has a unique bottom without peaks and has no local symmetry with respect to $v_{1}$, then $\mathscr{B}_{t}(X)=\mathscr{B}_{t}(V)$ holds, and hence $\hat{X}(t ; \tau)=E\left[f(V(t+\tau)) \mid \mathscr{B}_{t}(V)\right]$ a.s. holds. Actually the value of $V(t, \omega)$ is determined almost surely by the 
observed data $\{X(s, \omega) ; s \leq t\}$ as follows

$$
V(t, \omega)=f_{j(t, \omega)}^{-1}(X(t, \omega))
$$

with $j(t, \omega)$ given by (2.4).

Proof. The assertion follows from (2.2) and the above discussions.

TheOREm 2.5. Let $f(v)$ be a piecewise monotone and piecewise differentiable function which does not have any local symmetry. Then $\mathscr{B}_{t}(X)=\mathscr{B}_{t}(V)$ holds and hence $\hat{X}(t ; \tau)=E\left[f(V(t+\tau)) \mid \mathscr{B}_{t}(V)\right]$.

Proof. Since $f(v)$ has no local symmetry, we can recognize the times when $V(s, \omega)$ passes through one of peaks (or bottoms), by the same reason as in the proof of Theorem 2.4. Hence $j(s, \omega)$ defined by (2.1), can be traced for $s \leq t$, if $j\left(s^{\prime}, \omega\right)$ is determined once at a certain time $s^{\prime}(\leq t)$. Therefore we can determine $\left|j\left(s_{1}, \omega\right)-j\left(s_{2}, \omega\right)\right|$ for any $s_{1}, s_{2}<t$, by the observed data $\{X(s, \omega) ; s \leq t\}$. By the condition (1.3), there exist $s_{1}$ and $\mathrm{s}_{2}$ such that $\left|j\left(s_{1}, \omega\right)-j\left(s_{2}, \omega\right)\right|=L, s_{1}<s_{2}$. Then $V\left(s_{1}, \omega\right)$ is in either $I_{0}$ or $I_{L}$. If $L$ is even, we can determine $j\left(s_{1}, \omega\right)$ by observing which does $X(s, \omega)$ pass a peak or a bottom firstly after $s_{1}$. If $L$ is odd, we can find a time $s_{3}\left(s_{1}<s_{3}<s_{2}\right)$ when $V(s, \omega)$ passes $v_{(L+1) / 2}$ and can see the interval containing $V(s, \omega)$ for each $s$ in a neighbourhood of $s_{3}$. Thus we can trace $j(s, \omega), s \leq t$. Obviously $V(s, \omega)=f_{j(s, \omega)}^{-1}(X(s, \omega))$ is $\mathscr{B}_{t}(X)$-measurable.

The local limit theorem (2.3) may seem a condition too strong. A weaker condition does work well. Suppose that there exists a positive continuous function $a(v)$ on $(\ell, r)$ admitting the following local limit theorem

$$
O^{r}(V, t, \omega) \equiv \varlimsup_{h \rightarrow 0} \frac{|V(t+h, \omega)-V(t, \omega)|}{\gamma(h)}=a(V(t, \omega)) \text { a.s. }
$$

Define an increasing function $\varphi(v)$ on $(\ell, r)$ by

$$
\varphi(v) \equiv \int_{v^{*}}^{v} \frac{d u}{a(u)}, \quad v \in(\ell, r)
$$

with a fixed $v^{*}$ in $(\ell, r)$. Put $\bar{\ell} \equiv \varphi(\ell+0), \bar{r} \equiv \varphi(r-0)$ and $g(w) \equiv f \circ \varphi^{-1}(w)$ for $w \in(\bar{\ell}, \bar{r})$. Since $\varphi(v)$ is one-to-one map from $(\ell, r)$ onto $(\bar{\ell}, \bar{r})$,

$$
W(t, \omega) \equiv \varphi(V(t, \omega)), \quad t \in R,
$$

satisfies $\mathscr{B}_{t}(W)=\mathscr{B}_{t}(V)$ and 


$$
X(t, \omega) \equiv g(W(t, \omega))
$$

Since

$$
\operatorname{Or}(W ; t, \omega) \equiv \varlimsup_{h \rightarrow 0} \frac{|W(t+h, \omega)-W(t, \omega)|}{\gamma(h)}=1 \text { a.s. }
$$

for any $t$, we can apply Theorem 2.4 and Theorem 2.5 to the new base process $W(t)$. Define $F_{j}(h)$ and $\eta\left(f, a ; v_{j-1}\right)$ by

$$
\begin{gathered}
F_{j}(h) \equiv \begin{cases}\left|f^{\prime}\left(f_{j}^{-1}(h)\right)\right| a\left(f_{j}^{-1}(h)\right) & \text { if } f_{j}^{-1}(h) \in D(f), \\
\infty & \text { otherwise, }\end{cases} \\
\eta\left(f, a ; v_{j}\right) \equiv \inf \left\{\left|h-f\left(v_{j}\right)\right| ; F_{j}(h) \neq F_{j-1}(h)\right\}
\end{gathered}
$$

for $i=1,2, \cdots, L$.

Theorem 2.6. Suppose that the base process $V(t)$ satisfies (2.5) and that $f(v)$ is piecewise differentiable and piecewise monotone with peaks and bottoms $v_{1}, \cdots, v_{L}$. If $\eta\left(f, a ; v_{j}\right)=0$ for every $j, 1 \leq j \leq L$, then $\mathscr{B}_{t}(X)=$ $\mathscr{B}_{t}(V)$ holds, and hence $\hat{X}(t ; \tau)=E\left[f(V(t+\tau)) \mid \mathscr{B}_{t}(V)\right]$. Moreover, the value of $V(t, \omega)$ can be determined in a similar way to Theorem 2.4 and Theorem 2.5 .

\section{§3. Diffusion processes}

In this section, we consider the case that the strictly stationary process $V(t)$ is a conservative one-dimensional diffusion process with natural boundaries $\ell$ and $r(\ell<r)$. We assume that $V(t)$ satisfies a stochastic differential equation

$$
d V(t)=a(V(t)) d B(t)+b(V(t)) d t
$$

with a suitable Brownian motion $B(t)$ and with $C^{1}$-coefficients $a(v)(>0)$ and $b(v)$ on $(\ell, r)$. Then we know that the distribution $d m(v)$ of $V(t)$ is the speed measure and that the speed measure $m$ and the scale measure $s$ are given by

$$
\left\{\begin{array}{c}
m(v) \equiv c \int_{v^{*}}^{v} \frac{2}{a^{2}(u)} \exp \left[\int_{v^{*}}^{u} \frac{2 b(w)}{a^{2}(w)} d w\right] d u \\
s(v) \equiv \frac{1}{c} \int_{v^{*}}^{v} \exp \left[\int_{v^{*}}^{u} \frac{-2 b(w)}{a^{2}(w)} d w\right] d u
\end{array}\right.
$$

with $a v^{*}$ in $(\ell, r)$ and a normalizing constant $c$ as 


$$
m(r-0)-m(\ell+0)=1 .
$$

Since $\ell$ and $r$ are natural boundaries, we have

$$
s(r-0)=-s(\ell+0)=\int^{r-0} s(v) d m(v)=-\int_{\ell+0} s(v) d m(v)=\infty .
$$

As well known, the semigroup $\left\{T_{t}^{V}\right\}$ of the process $V(t)$ is self adjoint in $L^{2}(d m)$ and the transition probability of $V(t)$ has a symmetric density with respect to the speed measure $m$ (see McKean [7]). The time reversed process $\tilde{V}(t)=V(-t)$ is also a diffusion process with the same semigroup, and hence $V(t)$ satisfies the time reversed stochastic differential equation

$$
\tilde{d} V(t)=a(V(t)) \tilde{d} \tilde{B}(t)-b(V(t)) d t,
$$

with a suitable Brownian motion $\tilde{B}(t)$. Here we denote by $\int_{s}^{t} \psi(r, \omega) \tilde{d} V(r)$ the time reversed stochastic integral

$$
\int_{s}^{t} \psi(r, \omega) \tilde{d} V(r) \equiv \lim _{n \rightarrow \infty} \sum_{s \leq r_{j} \leq t} \psi\left(r_{j+1}, \omega\right)\left(V\left(r_{j+1}, \omega\right)-V\left(r_{j}, \omega\right)\right)
$$

with $r_{j} \equiv j \cdot 2^{-n}$. Put $\gamma^{*}(h) \equiv\left[2|h| \log _{2}|h|^{-1}\right]^{1 / 2}$. Then we can prove that

$$
O^{*}(V ; t, \omega) \equiv \varlimsup_{h \rightarrow 0} \frac{V(t+h, \omega)-V(t, \omega)}{\gamma^{*}(h)}=a(V(t, \omega)) \text { a.s. }
$$

for any $t$ (see McKean [6] p. 57 Problem 3) and that

$$
\varlimsup_{t \rightarrow-\infty} V(t, \omega)=r, \lim _{t \rightarrow-\infty} V(t, \omega)=\ell \text { a.s. }
$$

(see Ikeda-Watanabe [3] Chapter VI §3). Thus we can apply Theorem 2.6. By using the strong Markov properties of $V(t)$ and its time reversed process $\tilde{V}(t)$, we can state more precise results (c.f. Lee [5]).

Proposition 3.1. Let $f(v)$ be a function in $L^{2}(d m)$. Then the best non-linear predictor of $X(t+\tau)=f(V(t+\tau))$ for the given data $\{X(s, \omega) \equiv$ $f(V(s, \omega)) ; s \leq t\}$ is given by

$$
\hat{X}(t ; \tau)=E\left[\left(T_{\tau}^{v} f\right)(V(t)) \mid \mathscr{B}_{t}(X)\right] \text { a.s. }
$$

Proof. By the Markov property, we have

$$
\hat{X}(t ; \tau)=E\left[E\left[f(V(t+\tau)) \mid \mathscr{B}_{t}(V)\right] \mid \mathscr{B}_{t}(X)\right]=E\left[\left(T_{\tau}^{v} f\right)(V(t)) \mid \mathscr{B}_{t}(X)\right] .
$$


Now, we suppose that $f(v)$ has a unique bottom without peaks. Let $\eta\left(f, a, v_{1}\right)$ be the number defined by (2.9). Firstly, we discuss the case that $\eta\left(f, a, v_{1}\right)<\max \{f(\ell+0), f(r-0)\}-f\left(v_{1}\right)$. Then we can find a decreasing sequence $\left\{h_{n}\right\}$ in the $G(f)$ defined in Section 2 such that $\lim _{n \rightarrow \infty} h_{n}=$ $\eta\left(f, a, v_{1}\right)+f\left(v_{1}\right)$. Define stopping times of the time reversed process $\{\tilde{V}(t)=V(-t)\}$ by

$$
\left\{\begin{array}{l}
T(t, h, \omega) \equiv \sup \{s \leq t ; X(s, \omega)=h\}, \\
T(\omega)=T\left(t,\left\{h_{n}\right\}, \omega\right) \equiv \sup _{n} T\left(t, h_{n}, \omega\right) .
\end{array}\right.
$$

For almost every $\omega$ in $A \equiv\left\{\omega ; T\left(t, f\left(v_{1}\right), \omega\right) \leq T(\omega)\right\}$, we have the dichotomy

$$
V(t, \omega)= \begin{cases}f_{0}^{-1}(X(t, \omega)) & \text { if } O^{*}(X, T(\omega), \omega)=F_{0}(X(T(\omega), \omega)) \\ f_{0}^{-1}(X(t, \omega)) & \text { if } \operatorname{Or}^{*}(X, T(\omega), \omega)=F_{1}(X(T(\omega), \omega))\end{cases}
$$

Thus we have

$$
\hat{X}(t ; \tau, \omega)=\left(T_{\tau}^{V} f\right)\left(f_{j}^{-1}(X(t, \omega))\right)
$$

if $O^{r^{*}}(V, T(\omega), \omega)=F_{j}(X(T(\omega), \omega))$ for a.e. $\omega \in A$. In the contrary case $T\left(t, f\left(v_{1}\right), \omega\right)>T(\omega)$, we can not determine the value of $V(t, \omega)$ by the observed data $\{X(s, \omega) ; s \leq t\}$. Under the condition $A^{c}$, we have an expression

$$
\hat{X}(t ; \tau, \omega)=\sum_{j=0,1} p_{j}(\omega)\left(T_{\tau}^{V} f\right)\left(f_{j}^{-1}(X(t, \omega))\right) \text { a.e. } \omega \in A^{c},
$$

where $p_{j}(\omega)$ is the conditional probability of the event $V(t) \in I_{j}$ under the conditions $A^{c}$ and $\mathscr{B}_{t}(X)$. Let us now calculate $p_{j}(\omega), j=0,1$. Let functions $\varphi(v)$ and $g(w)$ be as in Section 2 with $v^{*}=v_{1}$. Then $g(w)$ is symmetric on an interval $[-q, q]$ with $g(q)=\eta\left(f, a, v_{1}\right)+f\left(v_{1}\right)$. The process $W(t)=\varphi(V(t))$ is a conservative diffusion process with the natural boundaries $\bar{\ell}$ and $\bar{r}$. Moreover, it satisfies the stochastic differential equations.

$$
\left\{\begin{array}{l}
d W(t)=d B(t)+\beta(W(t)) d t, \\
\tilde{d} W(t)=\tilde{d} \tilde{B}(t)-\beta(W(t)) d t,
\end{array}\right.
$$

with $\beta(w) \equiv b\left(\varphi^{-1}(w)\right) / a\left(\varphi^{-1}(w)\right)-\frac{1}{2} a^{\prime}\left(\varphi^{-1}(w)\right) . \quad$ Put $\quad T^{*}(\omega) \equiv T\left(t, f\left(v_{1}\right), \omega\right)=$ $\sup \{s<t ; W(s, \omega)=0\}$. Then either $|W(s, \omega)|=W(s, \omega), T^{*}(\omega)<s \leq t$ or $|W(s, \omega)|=-W(s, \omega), T^{*}(\omega)<s \leq t$ holds. Therefore the following stochastic integral makes a sense and we have the relation 


$$
\int_{T^{*}(\omega)}^{t} \psi(s, \omega) \tilde{d}|W(s, \omega)|=\left\{\begin{array}{cc}
\int_{T^{*}(\omega)}^{t} \psi(s, \omega) \tilde{d} W(s, \omega) & \text { if } W(t, \omega)>0, \\
-\int_{T^{*}(\omega)}^{t} \psi(s, \omega) \tilde{d} W(s, \omega) & \text { if } W(t, \omega)<0,
\end{array}\right.
$$

or a $\widetilde{\mathscr{B}}_{t}(V)$-adapted $\psi(s, \omega)$ in a suitable class.

LEMMA 3.2. Let $p_{0}(\omega)$ and $p_{1}(\omega)$ be the conditional probabilities in 3.10). Then it holds that

$$
\begin{aligned}
p_{1}(\omega)= & \left\{1+\exp \left[\int_{T^{*}(\omega)}^{t}(\beta(|W(u)|)+\beta(-|W(u)|)) \tilde{d}|W(u)|\right.\right. \\
& +\frac{1}{2} \int_{T^{*}(\omega)}^{t}\left(\beta^{2}(-|W(u)|)-\beta^{2}(|W(u)|)\right) d u \\
& \left.\left.-2 \int_{-|W(t)|}^{|W(t)|} \beta(w) d w\right]\right\}^{-1} .
\end{aligned}
$$

nd $p_{0}(\omega)=1-p_{1}(\omega)$ on $A^{c}$.

Proof. Since $W(t)$ is a stationary Markov process satisfying (3.11), he density of the distribution of $W(t)$ is given by

$$
c^{\prime} \exp \left[\int_{0}^{w} 2 \beta(u) d u\right]
$$

rith a normalizing constant $c^{\prime}$, we have

$$
\frac{P(W(t)<0}{P(W(t)>0} \mid \frac{|W(t)|)}{|W(t)|)}=\exp \left[-\int_{-|W(t)|}^{|W(t)|} 2 \beta(u) d u\right] .
$$

In the set $A^{c}$, the data $X(s, \omega), T^{*}(\omega)<s \leq t$, determine the values $W(s, \omega) \mid, T^{*}(\omega)<s \leq t$. By the strong Markov property, $\{W(s, \omega), s \leq$ $\left.{ }^{*}(\omega)\right\}$ is independent of $\left\{W(s, \omega) ; T^{*}(\omega)<s \leq t\right\}$. Hence it is sufficient or a computation of $p_{1}(\omega)$ that we consider the $\sigma$-field $\mathscr{B}_{t}(W) \cap \widetilde{\mathscr{B}}_{T^{*}}(W)$. et $P_{w}(\cdot)$ be the conditional measure defined on $\mathscr{B}_{t}(W) \cap \widetilde{\mathscr{B}}_{T^{*}}(W)$ condiioned by $W(t)=w$. Define

$$
\begin{aligned}
M(\omega) & \equiv \exp \left[\int_{T^{*}}^{t} \beta(W(u)) \tilde{d} W(u)+\frac{1}{2} \int_{T^{*}}^{t} \beta^{2}(W(u)) d u\right], \\
Y(s, \omega) & \equiv W\left((t-s)^{\vee} T(\omega)^{\vee} T^{*}(\omega)\right)-W(t) \\
& =B\left((t-s)^{\vee} T^{\vee} T^{*}\right)-B(t)-\int_{t}^{(t-s)^{\vee} T^{\vee} T^{*}} \beta^{2}(W(u)) d u .
\end{aligned}
$$

'hen $\left(Y(s, \omega), P_{w}\right)$ is a stopped Brownian motion at $\{q-w,-q-w$, - $w$ \}, where $P_{W}=M P_{w}$ (see Ikeda-Watanabe [3], Chapter IV, §4). Since 
the measure of a Brownian motion is symmetric, we can conclude the assertion by (3.12).

TheOREM 3.3. (i ) If $\eta\left(f, a, v_{1}\right)=0$, then $\mathscr{B}_{t}(X)=\mathscr{B}_{t}(V)$, and hence $\hat{X}(t ; \tau)=\left(T_{\tau}^{V} f\right)(V(t))$. The value of $V(t, \omega)$ is determined by (3.11).

(ii) If $f\left(v_{1}\right)<\eta\left(f, a, v_{1}\right)+f\left(v_{1}\right)<\max \{f(\ell+0), f(r-0)\}$, then we have

$$
\hat{X}(t ; \tau, \omega)= \begin{cases}\left(T_{\tau}^{V} f\right)(V(t, \omega)) & \text { for a.e. } \omega \text { in } A, \\ \sum_{j=0,1} p_{j}(\omega)\left(T_{\tau}^{V} f\right)\left(f_{j}^{-1}(X(t, \omega))\right) & \text { for a.e. } \omega \text { in } A,\end{cases}
$$

where $A=\left\{\omega ; T^{*}(\omega) \leq T(\omega)\right\}$.

(iii) If $\eta\left(f, a, v_{1}\right)+f\left(v_{1}\right)=\max \{f(\ell+0), f(r-0)\}$, then

$$
\hat{X}(t ; \tau, \omega)=\sum p_{j}(\omega)\left(T_{\tau}^{V} f\right)\left(f_{j}^{-1}(X(t, \omega))\right) \text { a.e. } \omega .
$$

We now remark another interesting fact. Suppose that the generator $\mathscr{L}=\frac{1}{2} a^{2}(v) \partial / \partial v^{2}+b(v) \partial / \partial v$ of $V(t)$ has an eigenvalue $\lambda(<0)$ and a corresponding eigenfunction $f_{\lambda}(v)$ in $L^{2}(d m)$; that is,

$$
\left(T_{t}^{v} f_{\lambda}\right)(v)=e^{\lambda t} f_{\lambda}(v) .
$$

Then the best non-linear predictor of the transformed process $X(t)=$ $f_{\lambda}(V(t))$ is given by

$$
\hat{X}(t ; \tau)=e^{\lambda \tau} X(t)
$$

by virtue of Proposition 3.1 and (3.13). The expression (3.14) means that the best non-linear predictor coincides with the best linear predictor. If $V(t)$ is Ornstein-Uhlenbeck process, then Hermite polynomials serve the eigenfunctions (c.f. Lee [5]).

\section{$\S 4$. Processes with differetiable paths}

In Section 2, we have discussed the cases that the base processes satisfy a local limit theorem either (2.3) or (2.5). If a base process $V(t)$ has differentiable paths, then such a local limit theorem does not hold. However we may expect that the $N$-th derivative $V^{(N)}(t, \omega)$ satisfies the local limit theorem (2.3). Actually, we have many examples in Gaussian stationary processes.

We assume that $V(t, \omega)$ is $N$-times continuously differentiable $(N \geq 1)$ and its $N$-th derivative $V^{(v)}(t, \omega)$ satisfies

$$
O^{\prime}\left(V^{(N)} ; t, \omega\right) \equiv \varlimsup_{h \rightarrow 0} \frac{\left|V^{(N)}(t+h, \omega)-V^{(N)}(t, \omega)\right|}{\gamma(h)}=1 \text { a.s. }
$$


for any $t$. If $f(v)$ is $(N+1)$-times differentiable and piecewise monotone, the process $X(t)=f(V(t))$ has continuously $N$-times differentiable paths and its $N$-th derivative $X^{(v)}(t)$ satisfies the local limit theorem

$$
O^{\gamma}\left(X^{(r)}, t, \omega\right) \equiv \varlimsup_{h \rightarrow 0} \frac{\left|X^{(v)}(t+h, \omega)-X^{(v)}(t, \omega)\right|}{\gamma(h)}=\left|f^{\prime}(V(t))\right| \text { a.s. }
$$

for any $t$. In a similar manner to Theorems 2.4 and 2.5, we can show:

THEOREM 4.1. Suppose that $V(t)$ has continuously $N$-times differentiable paths satisfying (4.1) and that $f(v)$ is $(N+1)$-times differentiable and piecewise monotone $(N \geq 1)$. If $f(v)$ has no local symmetry, then $\mathscr{B}_{t}(X)=$ $\mathscr{B}_{t}(V)$ and $\hat{X}(t ; \tau)=E\left[f(V(t+\tau)) \mid \mathscr{B}_{t}(V)\right]$ hold. In particular, if $f(v)$ has a single bottom without any peaks, then the value of $V(t, \omega)$ is determined by the observed data $\{X(s, \omega) ; s \leq t\}$ as follows

$$
V(t, \omega)= \begin{cases}f_{0}^{-1}(X(t, \omega)) & \text { if } T_{0}(T, \omega) \geq T_{1}(t, \omega), \\ f_{1}^{-1}(X(t, \omega)) & \text { if } T_{0}(t, \omega)<T_{1}(t, \omega) .\end{cases}
$$

where $T_{j}(t, \omega), j=0,1$, are defined by

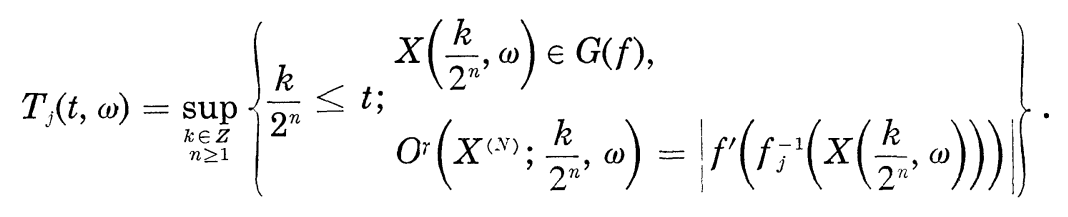

The theorem is merely a correspondence to the former result. But the following owes to the differentiability of paths essentially. We assume that

$$
P\left(\left\{\exists t \in R, V(t, \omega)=v^{*}, V^{\prime}(t, \omega)=0\right\}\right)=0 \quad \text { for any } v^{*}
$$

and that $f(v)$ is piecewise monotone with peaks and bottoms at $\left\{v_{1}, \cdots, v_{L}\right\}$. Put $C(f)=\left\{v ; \exists j, f\left(v_{j}\right)=f(v)\right\}$ and $\bar{C}(f)=\left\{f\left(v_{j}\right) ; 1 \leq j \leq L\right\}$. Then the set $\hat{Q}=\left\{\omega ; V(t, \omega)\right.$ is continuously differentiable, $\exists t \in R, V(t, \omega) \in C(f), V^{\prime}(t, \omega)$ $=0\}$ has probability one. For any $\omega$ in $\hat{\Omega}, X(t, \omega)$ does not take its maximal (or minimal) values in $\bar{C}(f)$ except the times when $V(t, \omega)$ passes through the points $\left\{v_{1}, \cdots, v_{L}\right\}$. Moreover, $V(t, \omega)$ does not turn its moving direction at the times. Thus we can have the time sequence when $V(s, \omega)$, $s \leq t$, moves into an interval $I_{j}$ from the other, by the observed data $\{X(s, \omega) ; s \leq t\}$. In the same way as proof of Theorem 2.5, we get the value of $j(s, \omega)$ defined by (2.1), if $L$ is even. If $L$ is odd, then we get an 
estimation $\hat{j}(s, \omega)$ such that

$$
j(s, \omega)= \begin{cases}\hat{j}(s, \omega) & \text { for } s \leq t, \quad \text { if } j(t, \omega) \geq(L+1) / 2, \\ L-\hat{j}(s, \omega) & \text { for } s \leq t, \quad \text { if } j(t, \omega)<(L+1) / 2 .\end{cases}
$$

Define

$$
\hat{V}(s, \omega) \equiv f_{\hat{\jmath}(s, \omega)}^{-1}(X(s, \omega)), \quad s \leq t .
$$

If $f(v)$ has a symmetry with respect to $v^{*}$, then the following dichotomy holds;

$$
V(s, \omega)= \begin{cases}\hat{V}(s, \omega) & \text { for } s \leq t, \quad \text { if } j(t, \omega) \geq(L+1) / 2, \\ 2 v^{*}-\hat{V}(s, \omega) & \text { for } s \leq t, \quad \text { if } j(t, \omega)<(L+1) / 2\end{cases}
$$

TheORem 4.2. Suppose that $V(t)$ has continuously $N$-times differentiable paths $(N \geq 1)$ and that $V(t)$ satisfies the condition (4.3). Suppose that $f(v)$ is piecewise monotone.

(i) If $L$ is even, then $\mathscr{B}_{t}(X)=\mathscr{B}_{t}(V)$ and $\hat{X}(t ; \tau)=E[f(V(t+\tau))]$ $\left.\mathscr{B}_{t}(V)\right]$ hold.

(ii) If $f(v)$ has a global symmetry with respect to a $v^{*}$, then the dichotomy (4.6) holds. Let $S(\omega)$ be a $\mathscr{B}_{t}(X)$-measurable random time such that $V(S(\omega), \omega) \neq 0, S(\omega)<t$. We have that

$$
\begin{aligned}
& \mathscr{B}_{t}(X)=\sigma\left(\left\{\left|V(s)-v^{*}\right| ; s \leq t\right\}\right) \quad \text { and } \\
& \mathscr{B}_{t}(V)=\mathscr{B}_{t}(X)^{\vee} \sigma(V(S(\omega), \omega)) .
\end{aligned}
$$

(iii) If $f(v)$ has no global symmetry and is $(N+1)$-times differentiable and if $V(t)$ satisfies (4.1), then we have $\mathscr{B}_{t}(X)=\mathscr{B}_{t}(V)$ and $\hat{X}(t ; \tau)=$ $E\left[f(V(t+\tau)) \mid \mathscr{B}_{t}(V)\right]$.

Proof. (i ) By the above discussion, we know $j(s, \omega)$, since $L$ is even. Therefore $V(s, \omega)$ is determined by $V(s, \omega)=f_{j(s, \omega)}^{-1}(X(s, \omega))$.

(ii) We have seen the dichotomy (4.6) whenever $f(v)$ has a global symmetry with respect to $v^{*}$. Therefore $\left|V(s, \omega)-v^{*}\right|=\left|\hat{V}(s, \omega)-v^{*}\right|$ is $\mathscr{B}_{s}(X)$-measurable. Thus we have the first equality. If we know once the actual value of $V\left(s^{\prime}, \omega\right)$ at a time $s^{\prime}<t$, then the dichotomy (4.6) determines the values of $V(s, \omega), s \leq t$.

(iii) If $f(v)$ has no global symmetry, we can find $i$ and $h$ such that $\left|f^{\prime}\left(f_{i}^{-1}(h)\right)\right| \neq\left|f^{\prime}\left(f_{L_{-i}}^{-1}(h)\right)\right|$. Appealing to (4.2), we can determine $j(s, \omega)$ at the time $s$ when $\hat{j}(s, \omega)=i$ and $X(s, \omega)=h$ hold. 


\section{§5. Stationary Gaussian processes}

In this section, we assume that process $V(t)$ is a stationary Gaussian process with continuous paths and that $V(t)$ has a canonical representation

$$
V(t)=\int_{-\infty}^{t} G(t-u) d B(u)
$$

with a suitable Brownian motion $\{B(t), t \in R\}$ and with a canonical kernel $G(t-u)$ (see Hida [2]). Let $\left\{T_{\tau}\right\}$ be the semi-group of the Brownian motion; that is,

$$
\left(T_{\tau} f\right)(v) \equiv \int_{-\infty}^{\infty} \frac{1}{\sqrt{2 \pi \tau}} f(u) \exp \left[-\frac{1}{2 \tau}(v-u)^{2}\right] d u
$$

Then we can easily see that

$$
\left\{\begin{array}{l}
\sigma^{2} \equiv E\left[V(t)^{2}\right]=\int_{0}^{\infty} G(u)^{2} d u \\
\hat{V}(t ; \tau) \equiv E\left[V(t+\tau) \mid \mathscr{B}_{t}(v)\right]=\int_{-\infty}^{t} G(t+\tau-u) d B(u) \\
\delta^{2} \equiv E\left[(V(t+\tau)-\hat{V}(t ; \tau))^{2} \mid \mathscr{B}_{t}(V)\right]=\int_{0}^{\tau} G(u)^{2} d u
\end{array}\right.
$$

For any $f(v)$ in $L^{2}\left(\left(2 \pi \sigma^{2}\right)^{-1 / 2} \exp \left[-v^{2} / 2 \sigma^{2}\right] d v\right), X(t)=f(V(t))$ is a strictly stationary process with mean $\left(T_{\sigma^{2}} f\right)(0)$ and with variance $\left(T_{\sigma^{2}} f^{2}\right)(0)$ $\left(T_{\sigma_{2}} f\right)(0)^{2}$. The best non-linear predictor of $X(t+\tau)$ for the given data $\{X(s, \omega) ; s \leqq t\}$ is expressed in the form

$$
\hat{X}(t ; \tau)=E\left[\left(T_{\delta 2} f\right)(\hat{V}(t ; \tau)) \mid \mathscr{B}_{t}(X)\right] .
$$

In particular, if $\mathscr{B}_{t}(X)=\mathscr{B}_{t}(V)$, then we have

$$
\hat{X}(t ; \tau)=\left(T_{\delta 2} f\right)(\hat{V}(t ; \tau)) .
$$

Since $V(t)$ has the canonical representation (5.1), $V(t)$ is ergodic. Hence we have that

$$
\begin{gathered}
\lim _{s \rightarrow \infty} \frac{1}{s} \int_{t-s}^{t} V(u, \omega) d u=0 \quad \text { a.s. } \\
\varlimsup_{s \rightarrow-\infty} V(s, \omega)=-\varliminf_{s \rightarrow-\infty} V(s, \omega)=\infty \quad \text { a.s. }
\end{gathered}
$$

Moreover, if $0<\varlimsup_{\lim _{h \rightarrow 0}}|V(t+h, \omega)-V(t, \omega)| / \gamma(h)<\infty$ a.s., then the value must be constant a.s. Therefore the assumption (2.5) is abandon. We can 
apply Theorem 2.4 and Theorem 2.5. Sufficient conditions for (2.3) have been discussed by many authors (for example, see Kōno [4]).

We now come to the case that the base process $V(t)$ has differentiable paths and that the $N$-th derivative of $V(t)$ satisfies the local limit theorem (2.3). In this case, we can apply Theorem 4.1. More interesting is the case that (4.3) is applied. Suppose that $f(v)$ is piecewise monotone and globally symmetric with respect to a $v^{*}$. As discussed in Section 4, we have the estimation $\{\hat{V}(s, \omega) ; s \leqq t\}$ with the dichotomy (4.6). Together with (5.5), we have the dichotomy

$$
\bar{v} \equiv \lim _{s \rightarrow \infty} \frac{1}{s} \int_{t-s}^{t} \hat{V}(u, \omega) d u=0 \text { or } 2 v^{*} \quad \text { a.s. }
$$

Hence we can determine $V(s, \omega)$ as follows

$$
V(s, \omega)= \begin{cases}\hat{V}(s, \omega) & \text { if } \bar{v}=0 \\ 2 v^{*}-\hat{V}(s, \omega) & \text { if } \bar{v}=2 v^{*}\end{cases}
$$

if $v^{*} \neq 0$. Thus we have $\mathscr{B}_{t}(X)=\mathscr{B}_{t}(V)$. If $v^{*}=0$, then either $\hat{V}(s, \omega)$ $=V(s, \omega)$ for $s \leqq t$, or $\hat{V}(s, \omega)=-V(s, \omega)$ for $s \leqq t$, holds. Since $\hat{V}(t ; \tau)$ is a linear functional of $\{V(s, \omega) ; s \leqq t\},|\hat{V}(t ; \tau)|$ is $\{\hat{V}(s, \omega) ; s \leqq t\}$-measurable; that is, $\mathscr{B}_{t}(X)$-measurable. Since $\left(T_{\tilde{\delta} 2} f\right)(v)$ is an even function, We have

$$
\hat{X}(t ; \tau)=\left(T_{\delta 2} f\right)(\hat{V}(t ; \tau))=\left(T_{\delta 2} f\right)(|\hat{V}(t ; \tau)|)
$$

with $\delta^{2}$ defined by (5.3).

Theorem 5.1. Suppose that a Gaussian stationary process $V(t)$ with the canonical representation (5.1) has $N$-times continuously differentiable paths and that the $N$-th derivative $V^{(N)}(t, \omega)$ satisfies the local limit theorem (2.3).

(i) If $f(v)$ is $(N+1)$-times continuously differentiable and piecewise monotone, then

$$
\hat{X}(t ; \tau)=\left(T_{\delta 2} f\right)(\hat{V}(t ; \tau)) \quad \text { with } \quad \delta^{2} \equiv \int_{0}^{\tau} G(u)^{2} d u .
$$

(ii) If $f(v)$ is piecewise monotone and globally symmetric, then (5.8) holds again.

For example, we now discuss double Markov, stationary Gaussian processes in the sense of Hida [2]. Such processes are classified in three cases by canonical representations: 
Case 1. $V(t)=\int_{-\infty}^{t}\left(b_{1} e^{-\alpha_{1}(t-u)}+b_{2} e^{-a_{2}(t-u)}\right) d B(u)$, with $a_{1} \neq a_{2}>0, b_{1}+b_{2} \neq 0,\left(a_{1} b_{2}+a_{2} b_{1}\right) /\left(b_{1}+b_{2}\right) \leqq 0$.

Case 2. $\quad V(t)=b \int_{-\infty}^{t}\left(e^{-a_{1}(t-u)}-e^{-a_{2}(t-u)}\right) d B(u)$ with $a_{1} \neq a_{2}>0, b \neq 0$.

Case 3. $V(t)=b \int_{-\infty}^{t}(t-u) e^{-a(t-u)} d B(u)$ with $a>0, b \neq 0$.

In Case 1, we can easily see that

$$
\varlimsup_{h \rightarrow 0} \frac{V(t+h, \omega)-V(t, \omega)}{\left|b_{1}+b_{2}\right| \gamma^{*}(h)}=1 \quad \text { a.s. for any } t,
$$

where $\gamma^{*}(h)=\left[2|h| \log _{2}(1 /|h|)\right]^{1 / 2}$. Thus we can apply Theorems 2.4 and 2.5. The quantities in (5.3) are calculated;

$$
\begin{aligned}
& \sigma^{2} \equiv E\left[V(t)^{2}\right]=\frac{b_{1}^{2}}{2 a_{1}}+\frac{2 b_{1} b_{2}}{a_{1}+a_{2}}+\frac{b_{2}^{2}}{2 a_{2}}, \\
& \hat{V}(t ; \tau)=\int_{-\infty}^{t}\left(b_{1} e^{-a_{1}(t+\tau-u)}+b_{2} e^{-a_{2}(t+\tau-u)}\right) d B(u), \\
& \delta^{2} \equiv\left(1-e^{-2 a_{1} \tau}\right) \frac{b_{0}^{2}}{2 a_{1}}+\left(1-e^{-\left(a_{1}+a_{2}\right) \tau}\right) \frac{2 b_{1} b_{2}}{a_{1}+a_{2}}+\left(1-e^{-2 a_{2} \tau}\right) \frac{b_{2}^{2}}{2 a_{2}} .
\end{aligned}
$$

In both Case 2 and Case 3, the process $V(t)$ has continuously differentiable paths. Moreover $\left(V(t), V^{\prime}(t)\right)$ is a two-dimensional diffusion process.

Case 2. The generator $\mathscr{L}$ is given by

$$
\mathscr{L}=\frac{1}{2} b^{2}\left(a_{2}-a_{1}\right)^{2} \frac{\partial^{2}}{\partial v_{2}^{2}}-\left(a_{1} a_{2} v_{1}+\left(a_{1}+a_{2}\right) v_{2}\right) \frac{\partial}{\partial v_{2}}+v_{2} \frac{\partial}{\partial v_{1}}
$$

and the $V(t)$ satisfies the stochastic differential equation;

$$
\left\{\begin{array}{l}
d V(t)=V^{\prime}(t) d t \\
d V^{\prime}(t)=b\left(a_{2}-a_{1}\right) d B(t)-a_{1} a_{2} V(t) d t-\left(a_{1}+a_{2}\right) V^{\prime}(t) d t .
\end{array}\right.
$$

The transition probability density $p\left(t, v_{1}, v_{2} ; u_{1}, u_{2}\right)$ is given by

$$
\left(4 \pi^{2} \operatorname{det} \Gamma(t)\right)^{-1 / 2} \exp \left[\frac{-1}{2 \operatorname{det} \Gamma(t)}\left\{\Gamma_{22}(t) \xi_{1}^{2}-2 \Gamma_{12}(t) \xi_{1} \xi_{2}+\Gamma_{11}(t) \xi_{2}^{2}\right\}\right],
$$

where $\Gamma(t)=\left(\Gamma_{i j}(t)\right)_{1 \leqq i, j \leqq 2}$, 


$$
\begin{aligned}
& \Gamma_{11}(t)=b^{2} \int_{0}^{t}\left(e^{-a_{1} u}-e^{-a_{2} u}\right)^{2} d u \\
& \Gamma_{12}(t)=\Gamma_{21}(t)=b^{2} \int_{0}^{t}\left(e^{-a_{1} u}-e^{-a_{2} u}\right)\left(-a_{1} e^{-a_{1} u}+a_{2} e^{-a_{2} u}\right) d u, \\
& \Gamma_{22}(t)=b^{2} \int_{0}^{t}\left(-a_{1} e^{-a_{1} u}+a_{2} e^{-a_{2} u}\right)^{2} d u \\
& \xi_{1}=u_{1}-\frac{a_{2} e^{-a_{1} t}-a_{1} e^{-a_{2} t}}{a_{2}-a_{1}} v_{1}-\frac{e^{-a_{1} t}-e^{-a_{2} t}}{a_{2}-a_{1}} v_{2}, \\
& \xi_{2}=u_{2}+\frac{a_{1} a_{2}\left(e^{-a_{1} t}-e^{-a_{2} t}\right)}{a_{2}-a_{1}} v_{1}+\frac{a_{1} e^{-a_{1} t}-a_{2} e^{-a_{2} t}}{a_{2}-a_{1}} v_{2} .
\end{aligned}
$$

As $t \rightarrow 0$, we have

$$
\begin{aligned}
& \Gamma_{11}(t)=\frac{b^{2}}{3}\left(a_{2}-a_{1}\right)^{2} t^{3}+O\left(t^{4}\right) \\
& \Gamma_{12}(t)=\Gamma_{21}(t)=\frac{b^{2}}{2}\left(a_{2}-a_{1}\right)^{2} t^{2}+O\left(t^{3}\right), \\
& \Gamma_{22}(t)=b^{2}\left(a_{2}-a_{1}\right)^{2} t+O\left(t^{2}\right) \\
& \operatorname{det} \Gamma(t)=\frac{b^{4}}{12}\left(a_{2}-a_{1}\right)^{4} t^{4}+O\left(t^{5}\right)
\end{aligned}
$$

Case 3. The generator $\mathscr{L}$ is given by

$$
\mathscr{L}=\frac{1}{2} b^{2} \frac{\partial^{2}}{\partial v_{2}^{2}}-\left(a^{2} v_{1}+2 a v_{2}\right) \frac{\partial}{\partial v_{2}}+v_{2} \frac{\partial}{\partial v_{1}}
$$

and the $V(t)$ satisfies the stochastic differential equation;

$$
\left\{\begin{array}{l}
d V(t)=V^{\prime}(t) d t \\
d V^{\prime}(t)=b d B(t)-a^{2} V(t) d t-2 a V^{\prime}(t) d t .
\end{array}\right.
$$

The transition probability density $p\left(t, v_{1}, v_{2} ; u_{1}, u_{2}\right)$ is given by the same formula as (5.10) with the replacement;

$$
\begin{aligned}
& \Gamma_{11}(t)=b^{2} \int_{0}^{t} u^{2} e^{-2 a u} d u, \\
& \Gamma_{12}(t)=\Gamma_{21}(t)=b^{2} \int_{0}^{t} u(1-a u) e^{-2 a u} d u, \\
& \Gamma_{22}(t)=b^{2} \int_{0}^{t}(1-a u)^{2} e^{-2 a u} d u, \\
& \xi_{1}=u_{1}-(a t+1) e^{-a t} v_{1}-t e^{-a t} v_{2}, \\
& \xi_{2}=u_{2}+a^{2} t e^{-a t} v_{1}-(1-a t) e^{-a t} v_{2} .
\end{aligned}
$$


Then their orders as $t \rightarrow 0$ are given by

$$
\begin{aligned}
& \Gamma_{11}(t)=\frac{b^{2}}{3} t^{3}+O\left(t^{4}\right), \quad \Gamma_{12}(t)=\Gamma_{21}(t)=\frac{b^{2}}{2} t+O\left(t^{3}\right), \\
& \Gamma_{22}(t)=b^{2} t+O\left(t^{2}\right) \quad \text { and } \quad \operatorname{det} \Gamma(t)=\frac{b^{4}}{12} t^{4}+O\left(t^{5}\right) .
\end{aligned}
$$

In both cases, we can show that the Green function

$$
g_{\alpha}\left(v_{1}, v_{2}, u_{1}, u_{2}\right)=\int_{0}^{\infty} p\left(t, v_{1}, v_{2} ; u_{1}, u_{2}\right) e^{-\alpha t} d t, \quad \alpha>0,
$$

is positive, continuous except on the diagonal $\left\{\left(v_{1}, v_{2}\right)=\left(u_{1}, u_{2}\right)\right\}$, and satisfies

$$
\mathscr{L} g_{\alpha}=\alpha g_{\alpha} \quad \text { for }\left(v_{1}, v_{2}\right) \neq\left(u_{1}, u_{2}\right)
$$

and

$$
\lim _{\left(v_{1}, v_{2}\right) \rightarrow\left(u_{1}, 0\right)} g_{\alpha}\left(v_{1}, v_{2}, u_{1}, 0\right)=\infty .
$$

Therefore we can apply Lemma 2.1 of Chapter 11 in Friedman [1]. Thus we can check in both Case 2 and Case 3, that $V(t)$ satisfies the condition (4.3).

By the proposition, we know that Theorem 5.1 is applicable to the double Markov, stationary Gaussian processes in the strict sense (Case 2 and Case 3).

TheOREm 5.2. Let $V(t)$ be a double Markov, stationary Gaussian process in the strict sense and let $f(v)$ be piecewise monotone.

(i) If $f(v)$ is 3-times continuously diffentiable, then

$$
\hat{X}(t ; \tau)=\left\{\begin{array}{lc}
\left(T_{\Gamma_{11}(\tau)} f\right)\left(\frac{a_{2} e^{-a_{1} \tau}-a_{1} e^{-a_{2 \tau} \tau}}{a_{2}-a_{1}} V(t)+\frac{e^{-a_{1 \tau}}-e^{-a_{2 \tau} \tau}}{a_{2}-a_{1}} V^{\prime}(t)\right) \\
& \text { for Case 2, } \\
\left(T_{\Gamma_{11}(\tau)} f\right)\left((1+a \tau) V(t)+\tau e^{-a_{\tau}} V^{\prime}(t)\right) & \text { for Case } 3 .
\end{array}\right.
$$

(ii) If $f(v)$ has a global symmetry with respect to a $v^{*}$ with $v^{*} \neq 0$, then (5.11) holds. If $f(v)$ has a global symmetry with respect to 0 , then (5.11) holds replacing $V(t, \omega)$ with $\hat{V}(t, \omega)$ defined by (4.5). 


\section{REFERENCES}

[1] Friedman, A., Stocastic differential equations and applications, Vol. 2, Academic Press, 1976.

[2] Hida, T., Canonical representation of Gaussian processes and their applications, Mem. Coll. Sci. Univ. Kyoto, 33 (1960), 109-155.

[ 3 ] Ikeda, N. and Watanabe, S., Stochastic differential equations and diffusion processes, North-Holland/Kodansha, 1981.

[4] Kōno, N., On the modulus of continuity of sample functions of Gaussian processes, J. Math. Kyoto Univ., 10-3 (1970), 493-536.

[5] Lee, Sheu-San, Non-linear prediction problems for Ornstein-Uhlenbeck process, Nagoya Math. J., 91 (1983), 173-183.

[6] McKean, H. P., Stochastic integrals, Academic Press, 1969.

[7] - Elementary solutions for certain parabolic partial differential equations, Trans. Amer. Math. Soc., 82 (1956), 519-548.

Izumi Kubo

Faculty of Integrated Arts and Sciences

Hiroshima University

Hiroshima, 730 Japan

Sheu-San Lee

Shenyang Chemical Engineering Institute

Shenyang

China 